Document downloaded from:

http://hdl.handle.net/10251/166895

This paper must be cited as:

Oser, D.; Pérez-Galacho, D.; Le Roux, X.; Tanzilli, S.; Vivien, L.; Labonte, L.; Cassan, E.... (2020). Silicon subwavelength modal Bragg grating filters with narrow bandwidth and high optical rejection. Optics Letters. 45(20):5784-5787. https://doi.org/10.1364/OL.394455

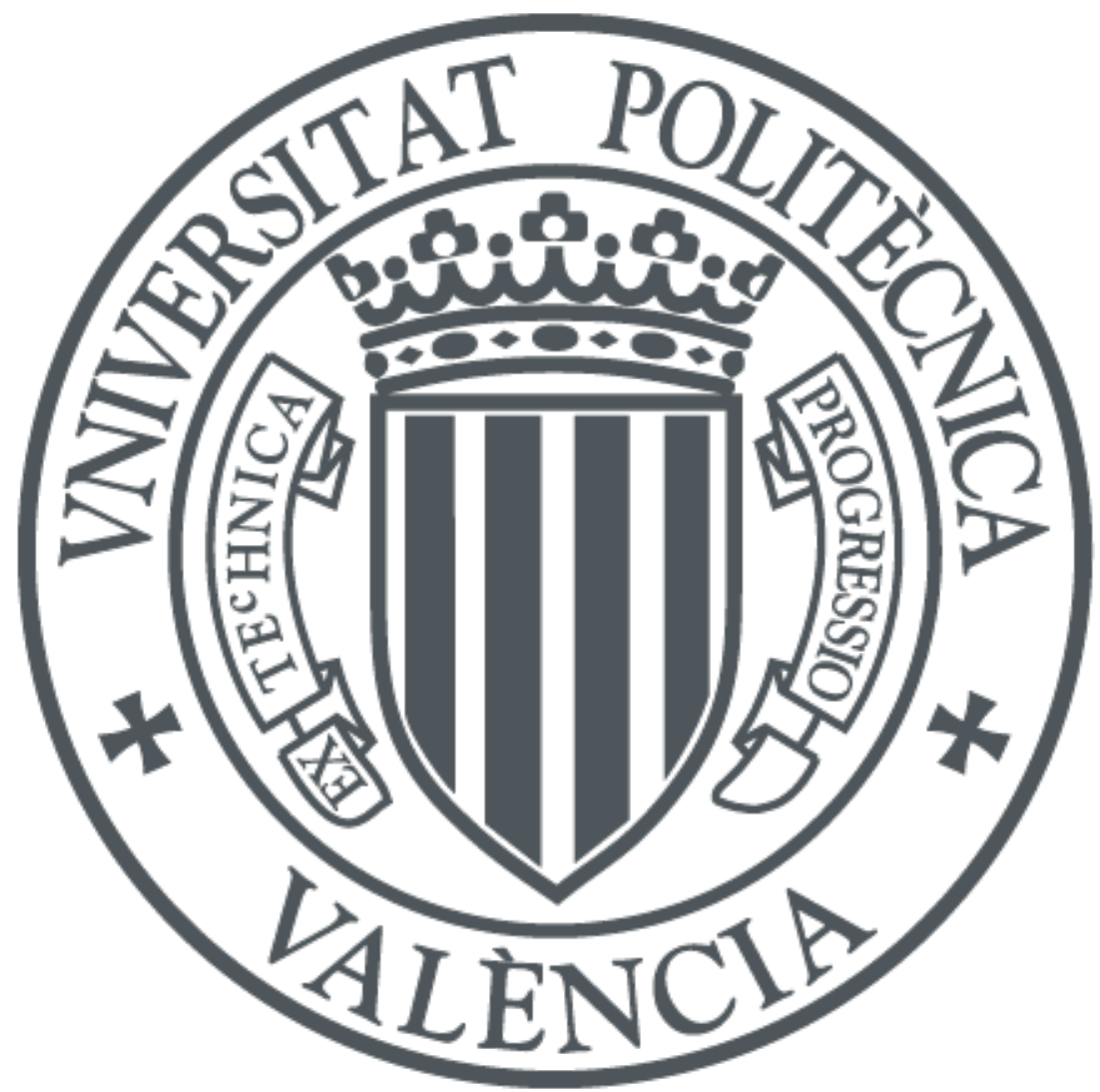

The final publication is available at

https://doi.org/10.1364/OL.394455

Copyright The Optical Society

Additional Information 


\title{
Silicon subwavelength modal Bragg grating filters with narrow bandwidth and high optical rejection
}

\author{
Dorian Oser, ${ }^{1, *}$ Diego Pérez-Galacho, ${ }^{2}$ Xavier Le Roux, ${ }^{3}$ Sébastien Tanzilli, ${ }^{3}$ \\ Laurent Vivien, ${ }^{1}$ Laurent Labonté, ${ }^{3}$ Éric Cassan, ${ }^{1}$ and Carlos Alonso-Ramos ${ }^{1}$ \\ ${ }^{1}$ Université Paris-Saclay, CNRS, Centre de Nanosciences et de Nanotechnologies, 91120, Palaiseau, France. \\ ${ }^{2}$ ITEAM research institute, Universitat Politècnica de València, Camino de Vera s/n, 46022, Valencia, Spain. \\ ${ }^{3}$ Université Côte d'Azur, CNRS, Institut de Physique de Nice (INPHYNI), Parc Valrose, 06108 Nice Cedex 2, France.
}

(Dated: December 4, 2020)

\begin{abstract}
Waveguide Bragg grating filters with narrow bandwidth and high optical rejection are key functions for several advanced silicon photonics circuits. Here, we propose and demonstrate a new Bragg grating geometry that provides narrowband and high rejection response. It combines the advantages of subwavelength and modal engineering. As a proof-of-concept demonstration, we implement the proposed Bragg filters in the 220-nm-thick Si technology with a single etch step. We experimentally show flexible control of the filter selectivity, with measured null-to-null bandwidths below $2 \mathrm{~nm}$, and strength of $60 \mathrm{~dB}$ rejection with a null-to-null bandwidth of $1.8 \mathrm{~nm}$.
\end{abstract}

Keywords: Quantum communication, Continuous variables, Nonlinear integrated photonics, Lithium niobate

\section{INTRODUCTION}

The silicon-on-insulator (SOI) platform is poised to deliver high-performance photonic circuits that can be fabricated in existing industrial facilities. Still, the realization of high rejection wavelength filters with narrow bandwidth remains a challenge. Such filters would be essential, for example, in silicon photon-pair sources exploiting spontaneous four-wave mixing (SFWM) process in Si micro-ring resonators [1-3]. Silicon photon-pair sources have a great potential for applications in quantum key distribution [4] and optical quantum computing [5]. However, integrated sources based on SFWM require on-chip rejections exceeding $100 \mathrm{~dB}$ over a small bandwidth $(\sim 2 \mathrm{~nm})$ due to the strong pump power compared to that of the photon-pairs $[6,7]$.

A myriad of optical filters has been reported for the silicon photonics technology, including Bragg grating filters $[8,9]$, cascaded micro-resonators $[10,11]$ and MachZehnder interferometers [12, 13]. Cascading several micro-ring resonators or Mach-Zehnder interferometers allowed the demonstration of remarkably large rejections $[6,14]$. However, in most cases these solutions require an active control of each element, and none of them showed a narrowband notch response. On the other hand, there have been remarkable achievements in the improvement of the performance of Si Bragg gratings in terms of selectivity, showing bandwidth below $1 \mathrm{~nm}[8,9,15-17]$ or rejection, with values exceeding $80 \mathrm{~dB}$ [18]. Subwavelength engineering of the grating lattice has shown to be a simple and powerful tool to achieve narrowband notch responses $[8,15,17]$. By exploiting metamaterial index engineering of the Bragg lattice, subwavelength-based filters implement effectively weak perturbations and thus narrowband responses with feature sizes larger than 50$100 \mathrm{~nm}$. Still, these weak filters require larger lengths

* dorian.oser@c2n.upsaclay.fr to achieve a given rejection level, which hinders maintaining the phase over the structure. Thus, the achievable rejection level in these filters is limited to $\sim 40 \mathrm{~dB}$ due to phase errors arising from fabrication imperfections. Concurrently, we have recently shown that noncoherent cascading of multi-modal waveguide Bragg gratings allows ultra-high rejection levels, exceeding $80 \mathrm{~dB}$ [18]. The idea lies in implementing several filter sections with Bragg gratings that couple back-reflections into a high-order waveguide mode, and connect them by singlemode waveguides that radiate back-reflections propagating in a high-order mode. By avoiding coherent interaction among filters, we achieve efficient cascading even in the presence of phase errors. A conceptually similar approach has been implemented, based on the cascading of contra-directional gratings that couple back-reflections into a different waveguide $[19,20]$. Nevertheless, these contradirectional couplers exhibited wideband notch responses, of $6 \mathrm{~nm}$ bandwidth at $1550 \mathrm{~nm}$. Sub-nanometer bandwidth has been achieved with single-stage contradirectional couplers in rib waveguides [21]. However, these filters required two etch steps, complicating fabrication, and have not been cascaded. Multi-modal-based multistage filters with a single etch step showed a bandwidth of $7 \mathrm{~nm}$ at $10 \mathrm{~dB}$ rejection. The high index contrast in silicon makes it difficult to further reduce this bandwidth [18]. Indeed, achieving the $<10 \mathrm{~nm}$ bandwidth with a single etch already required the implementation of very narrow corrugations of 20-50 nm, at the limit of the fabrication capabilities of the silicon photonics technology $[18,20]$.

Here, we propose and experimentally demonstrate a new Bragg grating geometry that gathers the advantages of subwavelength engineering and non-coherent multimodal cascading to implement narrowband notch filters with high-rejection levels. In the proposed geometry, depicted in Fig. 1(a), the Bragg unit cell comprises two slightly different subwavelength sub-periods, implementing a differential approach that yields weak perturbations 
even with large corrugation widths. In addition, the corrugations in the left- and right-hand sides of the waveguide are shifted by half-a-period to enable coupling of the Bragg back-reflections into the antisymmetric first order mode [22]. This way, different filter sections can be interconnected with single-mode waveguides achieving non-coherent cascading [18].

Provided the grating has the proper geometrical symmetry, Bragg back-reflections can be coupled to a higher order-mode [22]. The Bragg resonance wavelength resulting in coupling between the forward-propagating fundamental mode and the backward-propagating first-order mode, $\lambda_{o}$, is determined by the phase-matching condition, $\lambda_{o}=\Lambda\left(n_{\text {eff }}^{(1)}+n_{\text {eff }}^{(2)}\right) / p$. Here, $\Lambda$ is the grating period, $p$ is the Bragg order, while $n_{e f f}^{(1)}$ and $n_{\text {eff }}^{(2)}$ are the effective indexes of the fundamental and first-order modes, respectively. The rejection level $(R)$ and the notch bandwidth $(\Delta \lambda)$ of the filter can be theoretically estimated from coupled-mode theory (CMT) as $R=\tanh ^{2}\left(\kappa L_{F}\right)$ and

$$
\Delta \lambda=\frac{\lambda_{0}^{2}}{\pi n_{g}} \sqrt{\kappa^{2}+\frac{\pi^{2}}{L_{F}^{2}}},
$$

with $L_{F}$ the filter length, $n_{g}$ the group index and $\kappa$ the coupling coefficient [23].

In the proposed geometry, the grating lattice is defined by the waveguide width $\left(W_{w g}\right)$, the minimum corrugation depth $\left(W_{c o r r}\right)$, the corrugation difference $(d W)$, the lengths of the teeth $\left(L_{T}\right)$ and gap $\left(L_{G}\right)$ and the period $\left(\Lambda=2 L_{T}+2 L_{G}\right)$.

The Bragg coupling strength is mainly determined by the corrugation difference, $d W$, between the two subwavelength corrugations, rather than by the minimum corrugation depth $\left(W_{\text {corr }}\right)$, see Fig. 1(a). Thus, we have a large flexibility in choosing the minimum corrugation width. On the other hand, the corrugation on each side of the waveguide is shifted half-a-period to allow excitation of symmetric and anti-symmetric modes [22].

We designed the Bragg grating to operate with transverse-electric (TE) polarized light, considering coupling between the fundamental $\left(T E_{0}\right)$ and first-order $\left(T E_{1}\right)$ modes at a wavelength close to $1550 \mathrm{~nm}$. The proposed design has a silicon thickness of $220 \mathrm{~nm}$ and air as upper-cladding material. The waveguide width is $W_{w g}=1150 \mathrm{~nm}$, the minimum corrugation is $W_{\text {corr }}=$ $100 \mathrm{~nm}$, and the period is $\Lambda=290 \mathrm{~nm}$. In the subwavelength sub-period, the gaps have a length of $L_{G}=70 \mathrm{~nm}$ and the teeth have a length of $L_{T}=75 \mathrm{~nm}$. The minimum feature in the filter is determined by the gap length, of $L_{G}=70 \mathrm{~nm}$. This feature is compatible with electron-beam lithography and advanced optical immersion lithography techniques [24].

To illustrate the multi-modal nature of the proposed grating, we have calculated the transmission along a $100-\mu \mathrm{m}$-long filter using 3D finite-difference time domain (FDTD) simulations [25]. We chose this length to limit the calculation time. As excitation we used the fundamental TE mode of a $1150 \mathrm{~nm}$ wide strip waveguide. The field distribution of the transmitted and reflected light near $1550 \mathrm{~nm}$ wavelength (within the Bragg notch) are shown in Fig. 1(b) and Fig. 1(c), respectively. As expected, the transmitted light remains in the fundamental mode, while the back-reflected light is carried by the first-order mode.

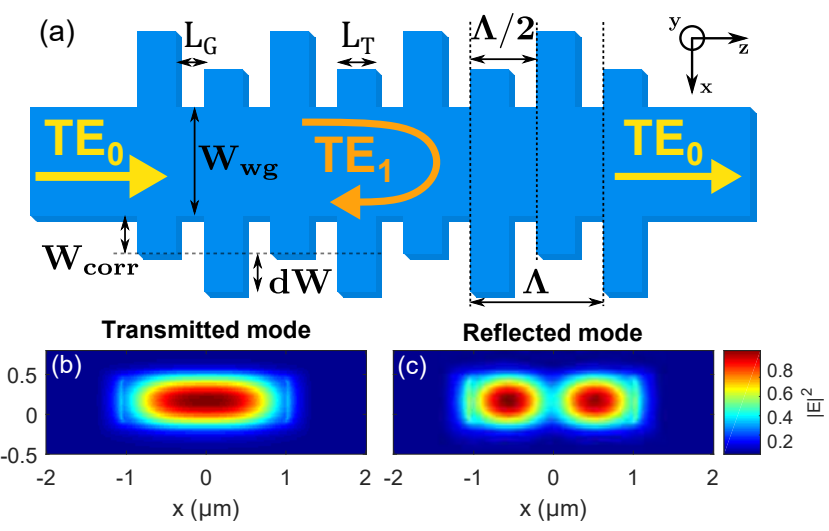

FIG. 1. (a) Schematic view of the proposed subwavelength multi-modal Bragg grating filter. (b) Mode profile of the injected/transmitted mode, (c) mode profile of the reflected mode, both at a wavelength of $1550 \mathrm{~nm}$.

We compare the bandwidth of the proposed subwavelength grating with that of an asymmetric filter implemented with a conventional rectangular corrugation [18]. We considered a corrugation width of $50 \mathrm{~nm}$, waveguide width of $1100 \mathrm{~nm}$, a pitch of $290 \mathrm{~nm}$ and a duty cycle of $50 \%$. The simulated transmission spectra are shown in Fig. 2(a). We can see a substantial reduction in rejection $(\sim 70 \%)$ and bandwidth in the case of the subwavelength filter. Still, the bandwidth reduction is less apparent for this filter length. To better show the bandwidth reduction provided by the subwavelength corrugation, we calculated the bandwidth as a function of the length, using Eq. (1). The values of group index $\left(n_{g}\right)$ and coupling coefficient $(\kappa)$ were extracted from the simulations in Fig. $2 \mathrm{~b}$ then used in Eq. (1) to predict the filter behavior [8]. As shown in Fig. 2(b), when the filter length increases, the subwavelength geometry yields up to a two-fold reduction in the notch bandwidth.

\section{EXPERIMENTAL DEMONSTRATION}

To evaluate the performance of the proposed asymmetric subwavelength engineered geometry, we fabricated different Bragg filters in the SOI platform with $220 \mathrm{~nm}$ thick Si and $3 \mu \mathrm{m}$ thick buried oxide layer (see Fig. 3). The filters were fabricated using electron beam lithography (Nanobeam NB-4 system, $80 \mathrm{kV}$ ) with $5 \mathrm{~nm}$ stepsize, followed by a dry etching process with an inductively coupled plasma etcher (SF6 and C4f8 gas) to pattern the structures. Experimentally, light was injected 

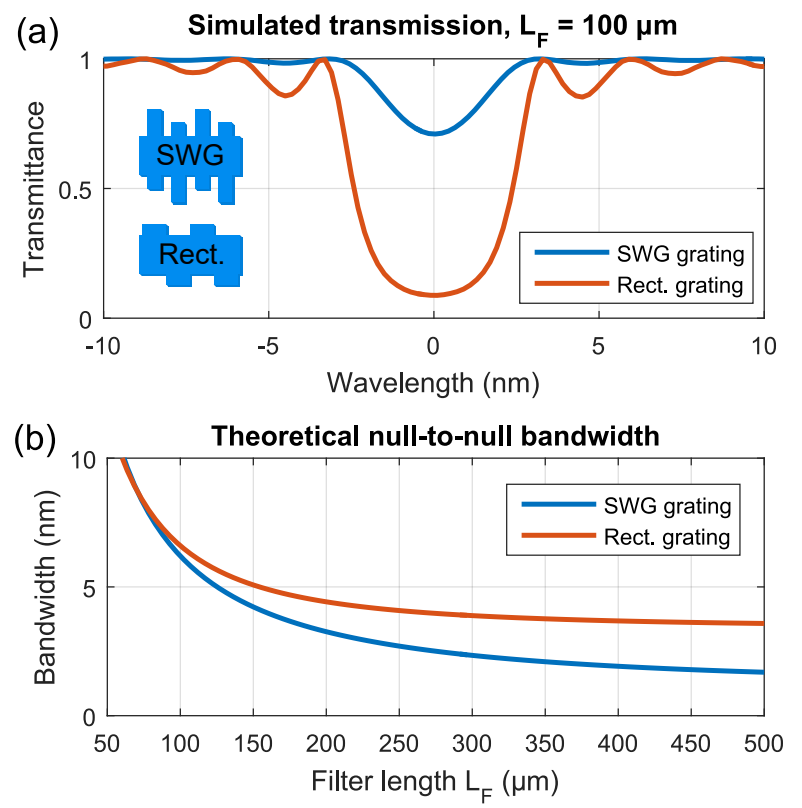

FIG. 2. (a) Simulated transmission of a Bragg filter with asymmetrical rectangular corrugation and proposed subwavelength approach. They exhibit null-to-null bandwidth of 6.6 and $6.2 \mathrm{~nm}$, respectively. (b) Theoretical null-to-null bandwidth extracted from the FDTD simulation, the group index $n_{g}$ values being 3.95 and 4.13 for the SWG and rectangular grating, respectively.

and extracted through the chip surface using subwavelength engineered grating couplers [26, 27] and cleaved single mode (SMF-28) optical fibers. We set a filter length of $500 \mu \mathrm{m}$, and varied the corrugation difference, $d W$, between $20 \mathrm{~nm}$ and $100 \mathrm{~nm}$. The minimum corrugation width in the filter is $120 \mathrm{~nm}\left(W_{\text {corr }}+d W=\right.$ $100 \mathrm{~nm}+20 \mathrm{~nm}$ ). We also used two waveguide widths $W_{w g}=1150 \mathrm{~nm}$ and $1200 \mathrm{~nm}$. The input and output fiber-chip couplers were connected to single-mode strip waveguides. We included $20 \mu \mathrm{m}$-long adiabatic tapers between the 400-nm-wide single-mode waveguide and a 1150-nm-wide multimode strip waveguide before and after the filters. To reduce the insertion losses of the grating, we implemented a smooth transition at the beginning and end of the gratings. The minimum corrugation width $\left(W_{\text {corr }}\right)$ was linearly varied between $50 \mathrm{~nm}$ and $100 \mathrm{~nm}$ over 20 periods, while implementing no differential corrugation, i.e. $d W=0 \mathrm{~nm}$. Note that for $d W=0 \mathrm{~nm}$, the two grating sub-periods are identical. Then the effective period in the transition, of $\Lambda / 2=145 \mathrm{~nm}$, lies in the subwavelength regime.

In Fig. 4(a), we plot the measured bandwidth and rejection for the fabricated filters, with $d W=20-100 \mathrm{~nm}$ and $W_{w g}=1150-1200 \mathrm{~nm}$. We experimentally show that the bandwidth can be tuned between $1 \mathrm{~nm}$ and $2 \mathrm{~nm}$, just by changing the corrugation difference. The increasing waveguide width results in a red-shift of the resonances as the effective index of the modes increases, as well as a

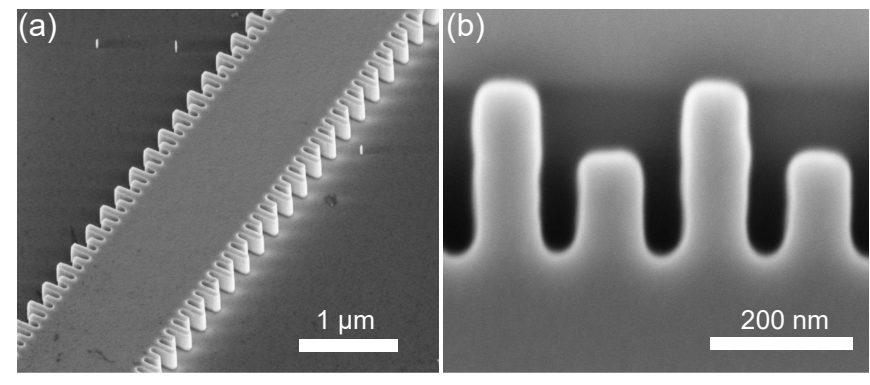

FIG. 3. Scanning electron microscope (SEM) images of (a) Bragg grating filter, and (b) detail of the differential corrugation geometry.

weaker rejection, as the perturbation is weakened. Naturally, it can be observed in Fig. 4(b) that low rejection is correlated with reduced bandwidth. The dotted line represents the bandwidth-rejection relation predicted using Eq. (1) from the coupled-mode theory. There is a very good agreement between coupled-mode theory and experiments. As an example, Fig. 4(b) shows the measured transmittance spectrum for three different filters with $d W=20 \mathrm{~nm}, d W=50 \mathrm{~nm}$ and $d W=100 \mathrm{~nm}$. As expected, wider corrugation differences result in wider and deeper rejection bands. Note that larger corrugations also yield higher (Bloch-Floquet) mode effective indices that redshift the center wavelength of the rejection band. These results demonstrate the great bandwidth flexibility enabled by the proposed geometry. For comparison, rectangular Si Bragg gratings with a corrugation of $50 \mathrm{~nm}$ (at the limit of conventional e-beam fabrication tools) yield a bandwidth near $10 \mathrm{~nm}$ [18].

To demonstrate the effective cascading of the proposed grating, we implement the multi-section filter schematically described in Fig. 5(a). Different filter sections are separated by S-bends, implemented with a single-mode waveguide. These S-bends radiate the backreflections propagating in the first-order mode, avoiding unwanted interferences that would limit achievable rejection [18]. We include a multimode-coupler between the input waveguide and the first Bragg grating (see Fig. $5(\mathrm{a})$ ), that couples back-reflected light, propagating in the $T E_{1}$ mode, into the fundamental mode of the adjacent reference waveguide [28]. This way we can monitor back-reflected light from the first Bragg grating.

The fabricated multi-stage filter comprises 9 Bragg grating sections with $d W=100 \mathrm{~nm}$ and length of $300 \mu \mathrm{m}$. Figure 5(b) shows the measured transmittance spectrum for the through and reference ports. From the response of the reference port we estimate a null-to-null bandwidth of $1.8 \mathrm{~nm}$. At the same time, the cascaded filter provides a rejection of at least $60 \mathrm{~dB}$ in the through port. That is almost $35 \mathrm{~dB}$ increase compared with the single-section device. Note that the 3dB-bandwidth is about $3.3 \mathrm{~nm}$ due to the misalignment of the side lobes of the different filters. This is half the $3 \mathrm{~dB}$ bandwidth of our previously reported cascaded Bragg filter [18]. This opens the 

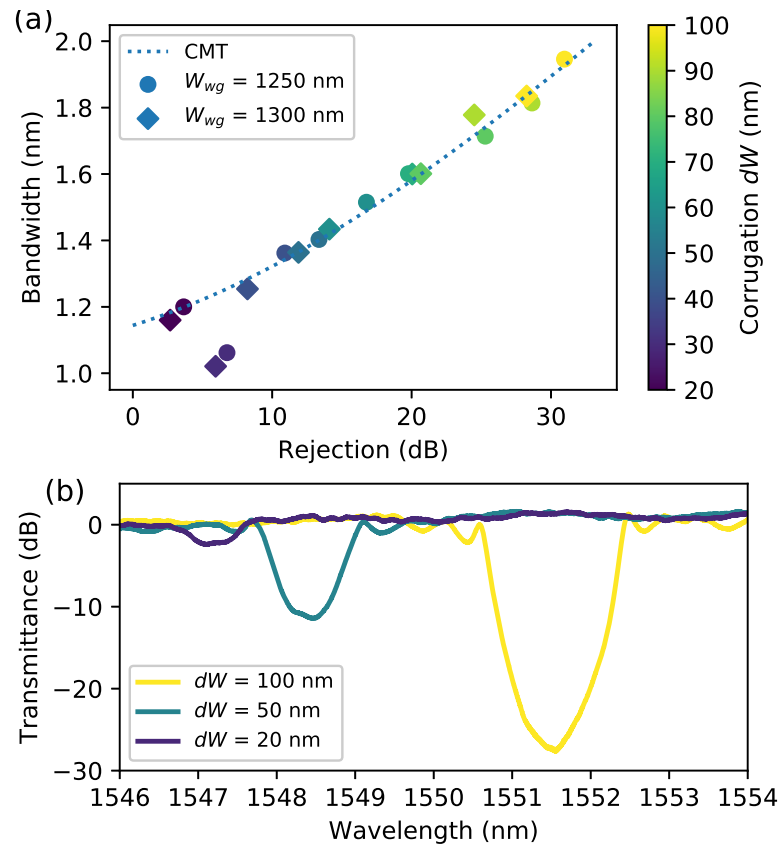

FIG. 4. (a) Measured null-to-null bandwidth as a function of the filter rejection for various $d W$ and fixed length of $500 \mu \mathrm{m}$, in doted line is the CMT prediction of bandwidth and rejection for the same filter length and a group index $n_{g}=4.2$. (b) Measured transmittance spectrum for three different corrugation width differences and $W_{w g}=1150 \mathrm{~nm}$.

possibility to exploit nearest resonances from the ring in photon-pair generation, which were previously discarded due to a too wide filter notch [7].

\section{CONCLUSION}

In conclusion, we have proposed and experimentally demonstrated a new waveguide Bragg grating geometry that exploits subwavelength structuration and noncoherent cascading to achieve flexible control in the notch bandwidth and strong optical rejection, simultaneously. Subwavelength engineering of the Bragg unit cell leads to weak Bragg perturbations with comparatively large corrugation depths. On the other hand, the excitation of the counter-propagating first-order mode enables effective cascading of different Bragg grating sections to increase the notch rejection. We have implemented the proposed approach in the SOI technology with 220-nmthick guiding silicon layer and a single etch step. We have shown measured notch bandwidths below $2 \mathrm{~nm}$ with corrugation depths larger than $100 \mathrm{~nm}$, illustrating the flexibility in bandwidth control provided by our geometry. By exploiting non-coherent cascading, we have also demonstrated a remarkably large rejection exceeding $60 \mathrm{~dB}$ for a filter with a bandwidth below $2 \mathrm{~nm}$. These results show the potential of the proposed approach for the development of flexible and reliable Si Bragg filters, and opens
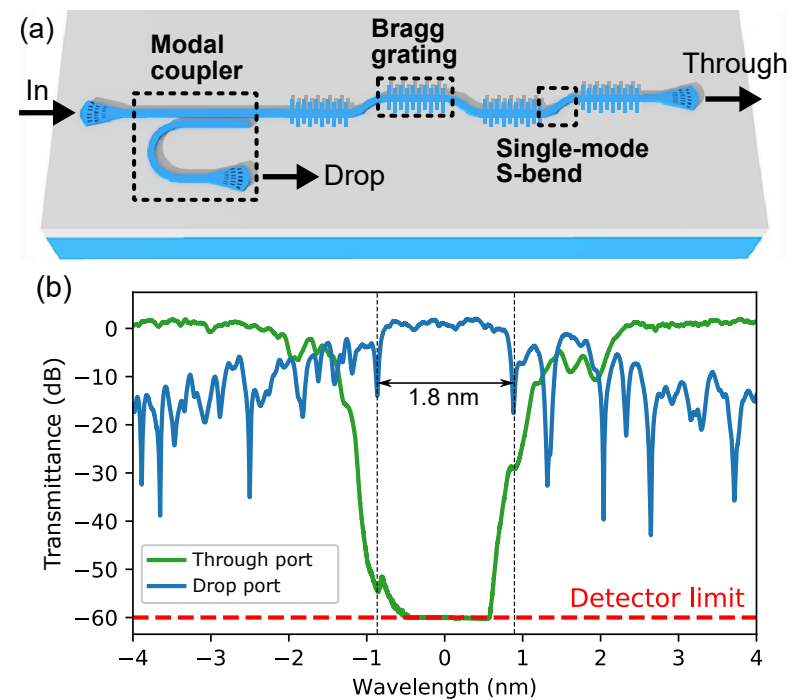

FIG. 5. (a) Schematic view of the circuit with a modal coupler and a cascaded Bragg filters. (b) Transmission spectra of a cascaded asymmetric subwavelength filter with a single section as reference. The cascaded filter is composed of 9 sections of $300 \mu \mathrm{m}$ long filter with a corrugation $W_{\text {corr }}$ and differential corrugation $d W$ of $100 \mathrm{~nm}$. The back reflected signal correspond to the back-reflection of the first filter section.

a new route for the implementation of high-performance Bragg grating filters harnessing both subwavelength and modal engineering. All while maintaining compatibility with standard phonics techniques.

\section{ACKNOWLEDGEMENT}

This work was partially funded by the Agence Nationale de la Recherche (ANR-SITQOM-15-CE240005, ANR-MIRSPEC-17-CE09-0041), the H2020 European Research Council (ERC POPSTAR 647342), and the French RENATECH network. The device fabrication was performed at the Plateforme de MicroNano-Technologie/C2N, partially funded by the Conseil Général de l'Essonne.

\section{DISCLOSURES}

The authors declare no conflicts of interest. 
[1] S. Azzini, D. Grassani, M. J. Strain, M. Sorel, L. Helt, J. Sipe, M. Liscidini, M. Galli, and D. Bajoni, Optics express 20, 23100 (2012).

[2] W. C. Jiang, X. Lu, J. Zhang, O. Painter, and Q. Lin, Optics Express 23, 20884 (2015).

[3] F. Mazeas, M. Traetta, M. Bentivegna, F. Kaiser, D. Aktas, W. Zhang, C. Ramos, L. Ngah, T. Lunghi, E. Picholle, et al., Opt. Express 24, 28731 (2016).

[4] N. Gisin, G. Ribordy, W. Tittel, and H. Zbinden, Reviews of modern physics 74, 145 (2002).

[5] E. Knill, R. Laflamme, and G. J. Milburn, Nature 409, 46 (2001).

[6] M. Piekarek, D. Bonneau, S. Miki, T. Yamashita, M. Fujiwara, M. Sasaki, H. Terai, M. G. Tanner, C. M. Natarajan, R. H. Hadfield, et al., Optics letters 42, 815 (2017).

[7] D. Oser, S. Tanzilli, F. Mazeas, C. Alonso-Ramos, X. Le Roux, G. Sauder, X. Hua, O. Alibart, L. Vivien, É. Cassan, et al., npj Quantum Information 6, 1 (2020).

[8] D. Pérez-Galacho, C. Alonso-Ramos, F. Mazeas, X. Le Roux, D. Oser, W. Zhang, D. Marris-Morini, L. Labonté, S. Tanzilli, E. Cassan, et al., Optics letters 42, 1468 (2017).

[9] C. Klitis, G. Cantarella, M. J. Strain, and M. Sorel, Optics letters 42, 3040 (2017).

[10] F. Xia, M. Rooks, L. Sekaric, and Y. Vlasov, Optics express 15, 11934 (2007).

[11] P. Dong, N.-N. Feng, D. Feng, W. Qian, H. Liang, D. C. Lee, B. Luff, T. Banwell, A. Agarwal, P. Toliver, et al., Optics express 18, 23784 (2010).

[12] Y. Ding, M. Pu, L. Liu, J. Xu, C. Peucheret, X. Zhang, D. Huang, and H. Ou, Optics Express 19, 6462 (2011).

[13] F. Horst, W. M. Green, S. Assefa, S. M. Shank, Y. A. Vlasov, and B. J. Offrein, Optics express 21, 11652 (2013).

[14] C. M. Gentry, O. S. Magaña-Loaiza, M. T. Wade, F. Pavanello, T. Gerrits, S. Lin, J. M. Shainline, S. D. Dyer, S. W. Nam, R. P. Mirin, et al., in CLEO: Applications and Technology (Optical Society of America, 2018), pp.
$\mathrm{JTh} 4 \mathrm{C}-3$.

[15] J. Wang, I. Glesk, and L. Chen, Electronics Letters 51, $712(2015)$.

[16] Z. Zou, L. Zhou, M. Wang, K. Wu, and J. Chen, Opt. Express 24, 12831 (2016).

[17] P. Cheben, J. Čtyrokỳ, J. H. Schmid, S. Wang, J. Lapointe, J. G. Wangüemert-Pérez, Í. Molina-Fernández, A. Ortega-Moñux, R. Halir, D. Melati, et al., Optics letters 44, 1043 (2019).

[18] D. Oser, F. Mazeas, X. Le Roux, D. Pérez-Galacho, O. Alibart, S. Tanzilli, L. Labonté, D. Marris-Morini, L. Vivien, É. Cassan, et al., Laser \& Photonics Reviews p. 1800226 (2019).

[19] X. Nie, N. Turk, Y. Li, Z. Liu, and R. Baets, Optics letters 44, 2310 (2019).

[20] M. Hammood, A. Mistry, M. Ma, H. Yun, L. Chrostowski, and N. A. Jaeger, Optics letters 44, 439 (2019).

[21] W. Shi, X. Wang, W. Zhang, L. Chrostowski, and N. Jaeger, Optics letters 36, 3999 (2011).

[22] H. Qiu, J. Jiang, P. Yu, T. Dai, J. Yang, H. Yu, and X. Jiang, Optics letters 41, 2450 (2016).

[23] A. Yariv, IEEE Journal of Quantum Electronics 9, 919 (1973).

[24] C. Dupre, C. Lapeyre, L. Adelmini, E. Arnoux, E. Guyez, P. Brianceau, L. Perraud, A. Fay, K. Hassan, Q. Wilmart, et al., in Photonics West (SPIE, 2020), pp. paper 1128413.

[25] Fdtd solutions, lumerical solutions, inc., http://www.lumerical.com.

[26] R. Halir, P. Cheben, S. Janz, D.-X. Xu, Í. MolinaFernández, and J. G. Wangüemert-Pérez, Optics letters 34, 1408 (2009).

[27] D. Benedikovic, P. Cheben, J. H. Schmid, D.-X. $\mathrm{Xu}$, B. Lamontagne, S. Wang, J. Lapointe, R. Halir, A. Ortega-Moñux, S. Janz, et al., Optics express 23, 22628 (2015).

[28] J. Wang, Y. Xuan, M. Qi, H. Huang, Y. Li, M. Li, X. Chen, Z. Sheng, A. Wu, W. Li, et al., Optics letters 40, 1956 (2015). 